\title{
Robust Stabilization of Hybrid Uncertain Stochastic Systems by Discrete-time Feedback Control
}

\author{
Yuyuan $\mathrm{Li}^{1}$, Jianqiu $\mathrm{Lu}^{2}$, Chunhai $\mathrm{Kou}^{3 * \dagger}$, Xuerong $\mathrm{Mao}^{2}$, Jiafeng $\mathrm{Pan}^{2}$ \\ ${ }^{1}$ College of Information Sciences and Technology, Donghua Univerisity, Shanghai 201620, China \\ ${ }^{2}$ Department of Mathematics and Statistics, University of Strathclyde, Glasgow G1 1XH, UK \\ ${ }^{3}$ Department of Applied Mathematics, Donghua Univerisity, Shanghai 201620, China
}

\section{SUMMARY}

This paper aims to stabilize hybrid stochastic differential equations (SDEs) with norm bounded uncertainties by feedback controls based on the discrete-time observations of both state and mode. The control structure appears only in the drift part (the deterministic part) of an SDE and the controlled system will be robustly exponentially stable in mean-square. Our stabilization criteria are in terms of linear matrix inequalities (LMIs) whence the feedback controls can be designed more easily in practice. An example is given to illustrate the effectiveness of our results. Copyright (c) 2010 John Wiley \& Sons, Ltd.

Received ...

KEY WORDS: Stochastic systems; Markovian jumping systems; uncertain systems; robust stabilization; discrete-time feedback control

\section{INTRODUCTION}

Stochastic systems have received a lot of attention as stochastic modeling has played a more and more important role in many branches of science and engineering in recent years (see e.g. [1-4]).

\footnotetext{
*Correspondence to: Chunhai Kou, Department of Applied Mathematics, Donghua Univerisity, Shanghai 201620, China.

†E-mail: kouchunhai@dhu.edu.cn

Copyright (C) 2010 John Wiley \& Sons, Ltd.
}

Prepared using ocaauth.cls [Version: 2010/03/27 v2.00] 
In practice, some stochastic systems, such as electric power systems, manufacturing systems and financial systems, may experience abrupt changes in their structures and parameters because of environment changes, random failures of components, etc. Hybrid stochastic differential equations (SDEs) (also known as SDEs with Markovian switching) have been used to model such systems (see e.g. [5-8]). An area of particular interest in the study of hybrid SDEs is the automatic control, with subsequent emphasis being placed on the stability analysis. There is an intensive literature in the area (see e.g. [9-18]). Particularly, Mao [10,11] are two of the most cited papers while Mao and Yuan [13] is the first book in this area.

It is well known that parameter uncertainties exist inherently in many dynamic systems and are frequently a cause of instability and performance degradation. Therefore, a great deal of attention has been devoted to the study of linear or nonlinear uncertain systems. In particular, the problem of robust stability and stabilization for uncertain systems has become an important issue and attracted an increasing interest (see e.g. [19-23] and references therein). Moon et al. [24] investigated the robust stabilization of uncertain state-delayed systems. A delay-dependent stabilization condition using a memoryless controller was formulated in terms of matrix inequalities. Based on a reduction method, a delayed feedback controller was designed in [25] to stabilize uncertain systems with time-varying delay. As for the stochastic cases, Lu et al. [26] considered the robust state-feedback stabilization of uncertain stochastic delay systems by applying the linear matrix inequality method. Wang et al. in [27] designed a state feedback controller to stabilize bilinear uncertain time-delay stochastic systems with Markovian jumping parameters in mean square sense. A robust delayedstate-feedback controller that exponentially stabilized uncertain stochastic systems was proposed in [28]. It is worth noting that the state feedback controllers in these papers require continuous observations of the system state for all time, which is expensive and impractical. Recently, for the sake of saving costs and being more realistic, Mao [29] studied the stabilization problem of hybrid stochastic systems by feedback control based on discrete-time state observations, which develops the corresponding studies for deterministic systems (see e.g. [30-32]). Then Mao et al. in [33] provided a better bound on the duration $\tau$ between two consecutive state observations and 
generalized the theory to a class of nonlinear hybrid stochastic systems, and You et al. [34] weakened the global Lipschitz assumption on coefficients and further investigated the asymptotic stabilization of nonlinear hybrid stochastic systems.

It is obserbed that the discrete-time feedback controls in [29, 33, 34] are based on the discretetime observations of the state but they still depend on the continuous-time observations of the mode. This is perfectly fine if the mode of the system is fully observable at no cost. However, the mode is not obvious in many real-world situations and it costs to identify the current mode of the system in practice. For example, Geromel $\mathbf{J}$ and Gabriel $\mathrm{G}$ [35] pointed out the necessity to design the feedback control based on discrete-time observations of both state and mode from the numerical point of view when studying the state feedback sampled-data control design problem of Markov jump systems. Therefore, it is necessary and reasonable that we identify the mode at the discrete times when we make observations for the state. These motivate us to go a step further to design a feedback controller $u(x(\delta(t)), r(\delta(t)))$, where $\delta(t)=[t / \tau] \tau$ for $t \geq 0$ and $\tau>0$, which is based on the discrete-time observations of both state and mode, to stabilize hybrid uncertain stochastic systems. It should be pointed out that our work is not a simple generalization of the existing results (see e.g. [29, 33, 34]). Mathematically speaking, the analysis in this paper becomes much more complicated due to the difficulties arisen from the discrete-time Markov chain $r([t / \tau] \tau)$. This is because that $r(t)$ is a jumping process (namely $r(t-)$ and $r(t+)$ may be at different states) and hence $r([t / \tau] \tau)$ dose NOT tend to $r(t)$ as $\tau$ tends to 0 , although $x([t / \tau] \tau)$ tends to $x(t)$. Therefore, the effect caused by $x([t / \tau] \tau)$ can be controlled in terms of $x(t)$ and $x(t)-x([t / \tau] \tau)$ but it is inappropriate to expect the effect caused by $r([t / \tau] \tau)$ is similar to that caused by $r(t)$ no matter how small $\tau$ is. These difficulties will be presented in detail later when we perform the proofs of our main results.

The organization of the rest of this article is as follows. After giving some notations at the end of this section, the main problem is stated in Section 2 and related definition and some useful lemmas are also given for later use. The main results are presented in Section 3. Section 4 covers a numerical example to demonstrate the main results in Section 3. Finally, the article is concluded in Section 5. 
Notation: For $x \in R^{n},|x|$ denotes its Euclidean norm. If $A$ is a vector or matrix, its transpose is denoted by $A^{T}$. For a matrix $A$, we let $|A|=\sqrt{\operatorname{trace}\left(A^{T} A\right)}$ be its trace norm and $\|A\|=\max \{|A x|$ : $|x|=1\}$ be the operator norm. If $A$ is a symmetric matrix $\left(A=A^{T}\right)$, denote by $\lambda_{\min }(A)$ and $\lambda_{\max }(A)$ its smallest and largest eigenvalue, respectively. For two symmetric matrices $A$ and $B$, $A>(<, \geq, \leq) B$ means that $A-B$ is positive definite (negative definite, positive semidefinite, negative semidefinite). The integer part of a real number $x$ will be denoted by $[x]$. Moreover, $\left(\Omega, \mathcal{F},\left\{\mathcal{F}_{t}\right\}_{t \geq 0}, \mathbb{P}\right)$ is a complete probability space with a filtration $\left\{\mathcal{F}_{t}\right\}_{t \geq 0}$ satisfying the usual conditions (i.e. it is increasing and right continuous with $\mathcal{F}_{0}$ containing all $\mathbb{P}$-null sets). Let $w(t)=\left(w_{1}(t), \cdots, w_{m}(t)\right)^{T}$ be an $m$-dimensional Brownian motion defined on the probability space. Denote by $L_{\mathcal{F}_{t}}^{2}\left(\mathbb{R}^{n}\right)$ the family of all $\mathcal{F}_{t}$-measurable $\mathbb{R}^{n}$-valued random variables $\xi$ such that $\mathbb{E}|\xi|^{2}<\infty$. Let $r(t), t \geq 0$, be a right-continuous Markov chain on the probability space taking values in a finite state space $S=\{1,2, \cdots, N\}$ with generator $\Gamma=\left(\gamma_{i j}\right)_{N \times N}$ given by

$$
\mathbb{P}\{r(t+\Delta)=j \mid r(t)=i\}= \begin{cases}\gamma_{i j} \Delta+o(\Delta) & \text { if } i \neq j, \\ 1+\gamma_{i i} \Delta+o(\Delta) & \text { if } i=j\end{cases}
$$

where $\Delta>0$ and $\gamma_{i j} \geq 0$ is the transition rate from $i$ to $j$ if $i \neq j$, while $\gamma_{i i}=-\sum_{j \neq i} \gamma_{i j}$. We assume that the Markov chain $r(\cdot)$ is independent of the Brownian motion $w(\cdot)$. It is known that almost all sample paths of $r(t)$ are step functions with a finite number of simple jumps in any finite subinterval of $R_{+}:=[0, \infty)$. We stress that almost all sample paths of $r(t)$ are right continuous. Let $C\left(\mathbb{R}^{n} \times \mathbb{R}_{+} ; \mathbb{R}_{+}\right)$denote the family of continuous functions from $\mathbb{R}^{n} \times \mathbb{R}_{+}$to $\mathbb{R}_{+}$, and $C^{2,1}\left(\mathbb{R}^{n} \times \mathbb{R}_{+} \times S ; \mathbb{R}_{+}\right)$denote the family of continuous functions $V(x, t, i)$ from $\mathbb{R}^{n} \times \mathbb{R}_{+} \times S$ to $\mathbb{R}_{+}$such that for each $i \in S, V(x, t, i)$ is continuously twice differentiable in $x$ and once in $t$.

\section{PRELIMINARIES AND PROBLEM STATEMENT}

Given an unstable hybrid uncertain stochastic system

$$
d x(t)=[A(r(t))+\Delta A(t, r(t))] x(t) d t+\sum_{k=1}^{m}\left[B^{k}(r(t))+\Delta B^{k}(t, r(t))\right] x(t) d w_{k}(t),
$$


on $t \geq 0$, with initial data

$$
x(0)=x_{0} \in L_{\mathcal{F}_{0}}^{2}\left(R^{n}\right), \quad r(0)=r_{0} \in S,
$$

where for any $i \in S, k=1,2, \cdots, m, A(i)=A_{i}$ and $B^{k}(i)=B_{i}^{k}$ are known constant matrices, while uncertainties $\Delta A(t, i), \Delta B^{k}(t, i)$ are assumed to be norm bounded, i.e.

$$
\Delta A(t, i)=L_{A} F_{A}(t) N_{i}, \quad \Delta B^{k}(t, i)=L_{B} F_{B}(t) E_{i}^{k}
$$

with known constant matrices $L_{A}, N_{i}, L_{B}, E_{i}^{k}$ and unknown matrix functions $F_{A}(t)$ and $F_{B}(t)$ having Lebesgue-measurable elements and satisfying

$$
F_{A}^{T}(t) F_{A}(t) \leq I, \quad F_{B}^{T}(t) F_{B}(t) \leq I, \quad \forall t \geq 0
$$

Now we aim to design a feedback control $u(x(\delta(t)), r(\delta(t)))$ based on the discrete-time observations of both state and mode in the drift part so that the controlled system

$$
\begin{aligned}
d x(t) & =[(A(r(t))+\Delta A(t, r(t))) x(t)+C(r(t)) u(x(\delta(t)), r(\delta(t)))] d t \\
& +\sum_{k=1}^{m}\left[B^{k}(r(t))+\Delta B^{k}(t, r(t))\right] x(t) d w_{k}(t)
\end{aligned}
$$

will be mean-square exponentially stable, where $C(i)=C_{i}$ is a given matrix while $u$ is a mapping from $\mathbb{R}^{n} \times S$ to $\mathbb{R}^{n}, \tau>0$ and

$$
\delta(t)=[t / \tau] \tau \text { for } t \geq 0
$$

in which $[t / \tau]$ is the integer part of $t / \tau$. We choose the structure control of the form $u(x, i)=K(i) x$ and then the controlled system (5) becomes

$$
\begin{aligned}
d x(t) & =[(A(r(t))+\Delta A(t, r(t))) x(t)+C(r(t)) K(r(\delta(t))) x(\delta(t))] d t \\
& +\sum_{k=1}^{m}\left[B^{k}(r(t))+\Delta B^{k}(t, r(t))\right] x(t) d w_{k}(t) .
\end{aligned}
$$

The equation (7) is in fact a special hybrid uncertain stochastic system with a bounded variable delay and the coefficients satisfy the Lipschitz condition and the linear growth condition. According to the existence-uniqueness theorem on SDDEs with Markovian switching (see e.g. [13]), equation (7) has a unique solution $x(t)$ under initial conditions (2) such that $\mathbb{E}|x(t)|^{2}<\infty$ for all $t \geq 0$. 
To close this section, we cite the following definition (see [13]) and two lemmas on matrix inequalities (see [36]) that will be in this paper.

\section{Definition 1}

The controlled system (7) with initial conditions (2) is said to be robustly exponentially stable in mean square, if there is a positive constant $\lambda>0$ such that for any uncertainties satisfying (3) and (4), the solution $x(t)$ satisfies

$$
\limsup _{t \rightarrow \infty} \frac{1}{t} \log \left(\mathbb{E}|x(t)|^{2}\right) \leq-\lambda
$$

\section{Lemma 1}

For any vectors $u \in \mathbb{R}^{q}, v \in \mathbb{R}^{l}$ and a matrix $M \in \mathbb{R}^{q \times l}$, the inequality

$$
2 u^{T} M v \leq r u^{T} M G M^{T} u+\frac{1}{r} v^{T} G^{-1} v
$$

holds for any symmetric positive definite matrix $G \in \mathbb{R}^{l \times l}$ and number $r>0$.

Lemma 2

Let A, B, D, F, W be matrices with suitable dimensions. If $W>0, F^{T} F \leq I$, then for any number $\varepsilon>0$ such that $W^{-1}-\varepsilon D D^{T}>0$, it holds that

$$
(A+D F B)^{T} W(A+D F B) \leq A^{T}\left(W^{-1}-\varepsilon D D^{T}\right)^{-1} A+\varepsilon^{-1} B^{T} B .
$$

\section{MAIN RESULTS}

Let us first present two lemmas in order to prove our main result.

Lemma 3

For any $t \geq 0, v>0$ and $i \in S$,

$$
P(r(s) \neq i \quad \text { for } \quad \text { some } \quad s \in[t, t+v] \mid r(t)=i) \leq 1-e^{-\bar{\gamma} v}
$$

where $\bar{\gamma}=\max _{i \in S}\left(-\gamma_{i i}\right)$. 


\section{Proof}

Given $r(t)=i$, define the stopping time

$$
\rho_{i}=\inf \{s \geq t: r(s) \neq i\}
$$

where and throughout this paper we set $\inf \emptyset=\infty$ (in which $\emptyset$ denotes the empty set as usual). It is well known (see [37]) that $\rho_{i}-t$ has the exponential distribution with parameter $-\gamma_{i i}$. Hence

$$
\begin{aligned}
& P(r(s) \neq i \quad \text { for some } \quad s \in[t, t+v] \mid r(t)=i) \\
= & P\left(\rho_{i}-t \leq v \mid r(t)=i\right)=\int_{0}^{v} \frac{1}{-\gamma_{i i}} e^{\gamma_{i i} s} d s \\
= & 1-e^{\gamma_{i i} v} \leq 1-e^{-\bar{\gamma} v}
\end{aligned}
$$

as desired.

\section{Lemma 4}

Let $x(t)$ be the solution of the uncertain hybrid SDE (7). Set

$$
\begin{gathered}
M_{A}=2 \max _{i \in S}\left(\left\|A_{i}\right\|^{2}+\left\|L_{A}\right\|^{2} \cdot\left\|N_{i}\right\|^{2}\right), \quad M_{C K}=\max _{i \in S}\left\|C_{i} K_{i}\right\|^{2}, \\
M_{B}=2 \max _{i \in S} \sum_{k=1}^{m}\left(\left\|B_{i}^{k}\right\|^{2}+\left\|L_{B}\right\|^{2} \cdot\left\|E_{i}^{k}\right\|^{2}\right),
\end{gathered}
$$

and define

$$
K(\tau)=\left(6 \tau^{2} M_{A}+6 \tau M_{B}+3 \tau^{2} M_{C K}\right) e^{6 \tau^{2} M_{A}+6 \tau M_{B}}
$$

for $\tau>0$. If $\tau$ is small enough for $2 K(\tau)<1$, then

$$
\mathbb{E}|x(t)-x(\delta(t))|^{2} \leq \frac{2 K(\tau)}{1-2 K(\tau)} \mathbb{E}|x(t)|^{2}
$$

holds for all $t \geq 0$.

\section{Proof}

Fix any integer $l \geq 0$. For $t \in[l \tau,(l+1) \tau)$, we have $\delta(t)=l \tau$. Then it follows from (7) that

$$
\begin{aligned}
x(t)-x(\delta(t)) & =x(t)-x(l \tau) \\
& =\int_{l \tau}^{t}[(A(r(s))+\Delta A(s, r(s))) x(s)+C(r(s)) K(r(l \tau)) x(l \tau)] d s \\
& +\sum_{k=1}^{m} \int_{l \tau}^{t}\left[B^{k}(r(s))+\Delta B^{k}(s, r(s))\right] x(s) d w_{k}(s) .
\end{aligned}
$$


By the fundamental inequality $|a+b+c|^{2} \leq 3|a|^{2}+3|b|^{2}+3|c|^{2}$, Hölder's inequality and Doob's martingale inequality, we can then derive

$$
\begin{aligned}
\mathbb{E}|x(t)-x(\delta(t))|^{2} \leq & 3 \mathbb{E}\left|\int_{l \tau}^{t}[A(r(s))+\Delta A(s, r(s))] x(s) d s\right|^{2} \\
& +3 \mathbb{E}\left|\int_{l \tau}^{t} C(r(s)) K(r(l \tau)) x(l \tau) d s\right|^{2} \\
& +3 \mathbb{E}\left|\sum_{k=1}^{m} \int_{l \tau}^{t}\left[B^{k}(r(s))+\Delta B^{k}(s, r(s))\right] x(s) d w_{k}(s)\right|^{2} \\
& \leq 3 \tau \int_{l \tau}^{t} \mathbb{E}\left(\|A(r(s))+\Delta A(s, r(s))\|^{2}|x(s)|^{2}\right) d s \\
& +3 \tau \int_{l \tau}^{t} \mathbb{E}\left(\|C(r(s)) K(r(l \tau))\|^{2}|x(l \tau)|^{2}\right) d s \\
& +3 \sum_{k=1}^{m} \int_{l \tau}^{t} \mathbb{E}\left(\left\|B^{k}(r(s))+\Delta B^{k}(s, r(s))\right\|^{2}|x(s)|^{2}\right) d s .
\end{aligned}
$$

Note that $\left\|F_{A}(t)\right\| \leq 1$ for any $t \geq 0$ from (4). Consequently, for any $i \in S$,

$$
\left\|A_{i}+\Delta A(t, i)\right\|^{2} \leq 2\left(\left\|A_{i}\right\|^{2}+\left\|L_{A} F_{A}(t) N_{i}\right\|^{2}\right) \leq M_{A}
$$

Similarly, for any $i \in S,\left\|B_{i}^{k}+\Delta B^{k}(t, i)\right\|^{2} \leq 2\left(\left\|B_{i}^{k}\right\|^{2}+\left\|L_{B}\right\|^{2}\left\|E_{i}^{k}\right\|^{2}\right)$, and then

$$
\sum_{k=1}^{m}\left\|B_{i}^{k}+\Delta B^{k}(t, i)\right\|^{2} \leq M_{B} .
$$

Substituting (11) and (12) together into (10), we have

$$
\begin{aligned}
\mathbb{E}|x(t)-x(\delta(t))|^{2} & \leq 3\left(\tau M_{A}+M_{B}\right) \int_{l \tau}^{t} \mathbb{E}|x(s)|^{2} d s+3 \tau^{2} M_{C K} \mathbb{E}|x(l \tau)|^{2} \\
& \leq 6\left(\tau M_{A}+M_{B}\right) \int_{l \tau}^{t} \mathbb{E}|x(s)-x(l \tau)|^{2} d s \\
& +\left(6 \tau^{2} M_{A}+6 \tau M_{B}+3 \tau^{2} M_{C K}\right) \mathbb{E}|x(l \tau)|^{2} .
\end{aligned}
$$

The well-known Gronwall inequality shows

$$
\mathbb{E}|x(t)-x(\delta(t))|^{2} \leq K(\tau) \mathbb{E}|x(l \tau)|^{2} \leq 2 K(\tau)\left(\mathbb{E}|x(t)-x(l \tau)|^{2}+\mathbb{E}|x(t)|^{2}\right),
$$

which implies (9) holds for $t \in[l \tau,(l+1) \tau)$. Then assertion (9) must hold for all $t \geq 0$ as $l \geq 0$ is arbitrary. 


\section{Theorem 1}

Assume that there exist positive definite matrices $Q_{i}$ and positive numbers $\rho_{i}, \varepsilon_{i}(i \in S)$ such that

$$
\begin{aligned}
\bar{Q}_{i}: & =Q_{i}\left(A_{i}+C_{i} K_{i}\right)+\left(A_{i}+C_{i} K_{i}\right)^{T} Q_{i}+\rho_{i} Q_{i} L_{A} L_{A}^{T} Q_{i}+\rho_{i}^{-1} N_{i}^{T} N_{i}+\sum_{j=1}^{N} \gamma_{i j} Q_{j} \\
& +\sum_{k=1}^{m}\left(B_{i}^{k}\right)^{T}\left(Q_{i}^{-1}-\varepsilon_{i} L_{B} L_{B}^{T}\right)^{-1} B_{i}^{k}+\varepsilon_{i}^{-1} \sum_{k=1}^{m}\left(E_{i}^{k}\right)^{T} E_{i}^{k}
\end{aligned}
$$

are all negative-definite matrices, and for any $i \in S$,

$$
Q_{i}^{-1}-\varepsilon_{i} L_{B} L_{B}^{T}>0
$$

Let $K(\tau)$ be the same as defined in Lemma 4 and set

$$
\begin{gathered}
\lambda_{M}=\max _{i \in S} \lambda_{\max }\left(Q_{i}\right), \quad \lambda_{m}=\min _{i \in S} \lambda_{\min }\left(Q_{i}\right), \quad \lambda=\max _{i \in S} \lambda_{\max }\left(\bar{Q}_{i}\right), \\
M_{Q C K}=\max _{i \in S}\left\|Q_{i} C_{i} K_{i}\right\|^{2}, \quad M_{C}=\max _{i \in S}\left\|C_{i}\right\|^{2}, \quad H_{K}=\max _{i, j \in S}\left\|K_{j}-K_{i}\right\|^{2}
\end{gathered}
$$

(and of course $\lambda<0$ ). If $\tau$ is sufficiently small for

$$
\lambda_{\tau}:=\lambda+2 \sqrt{\frac{2 M_{Q C K} K(\tau)}{1-2 K(\tau)}}+2 \lambda_{M} \sqrt{\frac{2 M_{C} H_{K}\left(1-e^{-\bar{\gamma} \tau}\right)}{1-2 K(\tau)}}<0,
$$

then the solution of (7) satisfies

$$
\limsup _{t \rightarrow \infty} \frac{1}{t} \log \left(\mathbb{E}|x(t)|^{2}\right) \leq \frac{\lambda_{\tau}}{\lambda_{M}}
$$

In other words, the hybrid uncertain SDE (7) is exponentially stable in mean square.

\section{Proof}

Let $V(x(t), r(t))=x^{T}(t) Q(r(t)) x(t)$, where $Q(i)=Q_{i}$ as $r(t)=i$. Applying the generalized Itô formula to $V$, we get

$$
d V(x(t), r(t))=\mathcal{L} V(x(t), r(t)) d t+d M_{1}(t)
$$

where $M_{1}(t)$ is a martingale with $M_{1}(0)=0$ and

$$
\begin{aligned}
\mathcal{L} V(x(t), i) & =2 x^{T}(t) Q_{i}\left[\left(A_{i}+\Delta A(t, i)\right) x(t)+C_{i} K(r(\delta(t))) x(\delta(t))\right]+\sum_{j=1}^{N} \gamma_{i j} x^{T}(t) Q_{j} x(t) \\
& +\sum_{k=1}^{m} x^{T}(t)\left(B_{i}^{k}+\Delta B^{k}(t, i)\right)^{T} Q_{i}\left(B_{i}^{k}+\Delta B^{k}(t, i)\right) x(t) .
\end{aligned}
$$


According to (3), (4) and Lemma 1, the first term can be treated as

$$
\begin{aligned}
& 2 x^{T}(t) Q_{i}\left(A_{i}+\Delta A(t, i)\right) x(t)+2 x^{T}(t) Q_{i} C_{i} K(r(\delta(t))) x(\delta(t)) \\
\leq & 2 x^{T}(t) Q_{i} A_{i} x(t)+\rho_{i} x^{T}(t)\left(Q_{i} L_{A} L_{A}^{T} Q_{i}\right) x(t)+\rho_{i}^{-1} x^{T}(t)\left(N_{i}^{T} N_{i}\right) x(t) \\
+ & 2 x^{T}(t) Q_{i} C_{i} K(r(\delta(t))) x(\delta(t)) \\
\leq & x^{T}(t)\left(Q_{i}\left(A_{i}+C_{i} K_{i}\right)+\left(A_{i}+C_{i} K_{i}\right)^{T} Q_{i}\right) x(t) \\
+ & \rho_{i} x^{T}(t)\left(Q_{i} L_{A} L_{A}^{T} Q_{i}\right) x(t)+\rho_{i}^{-1} x^{T}(t)\left(N_{i}^{T} N_{i}\right) x(t) \\
- & 2 x^{T}(t) Q_{i} C_{i} K_{i}(x(t)-x(\delta(t)))-2 x^{T}(t) Q_{i} C_{i}(K(r(t))-K(r(\delta(t)))) x(\delta(t))
\end{aligned}
$$

For the last term, we can, using (3) (4) and Lemma 2, show that

$$
\begin{aligned}
& \sum_{k=1}^{m} x^{T}(t)\left(B_{i}^{k}+L_{B} F_{B}(t) E_{i}^{k}\right)^{T} Q_{i}\left(B_{i}^{k}+L_{B} F_{B}(t) E_{i}^{k}\right) x(t) \\
\leq & \sum_{k=1}^{m} x^{T}(t)\left[\left(B_{i}^{k}\right)^{T}\left(Q_{i}^{-1}-\varepsilon_{i} L_{B} L_{B}^{T}\right)^{-1} B_{i}^{k}+\varepsilon_{i}^{-1}\left(E_{i}^{k}\right)^{T} E_{i}^{k}\right] x(t) .
\end{aligned}
$$

Substituting (17) and (18) into (16), we have that for any $i \in S$,

$$
\begin{aligned}
\mathcal{L} V(x(t), i) & \leq x^{T}(t) \bar{Q}_{i} x(t)-2 x^{T}(t) Q_{i} C_{i} K_{i}(x(t)-x(\delta(t))) \\
& -2 x^{T}(t) Q_{i} C_{i}(K(r(t))-K(r(\delta(t)))) x(\delta(t)) \\
& \leq \lambda|x(t)|^{2}+2 \sqrt{M_{Q C K}}|x(t)||x(t)-x(\delta(t))| \\
& -2 x^{T}(t) Q_{i} C_{i}(K(r(t))-K(r(\delta(t)))) x(\delta(t)) .
\end{aligned}
$$

Applying the generalized Itô formula now to $e^{\theta t} V(x(t), r(t))$, with $\theta=-\frac{\lambda_{\tau}}{\lambda_{M}}>0$, we then obtain that, for any $t \geq 0$,

$$
\begin{aligned}
e^{\theta t} x^{T}(t) Q(r(t)) x(t) & =x^{T}(0) Q(r(0)) x(0) \\
& +\int_{0}^{t} e^{\theta s}\left[\theta x^{T}(s) Q(r(s)) x(s)+\mathcal{L} V(x(s), r(s))\right] d s+M_{2}(t),
\end{aligned}
$$


where $M_{2}(t)$ is a continuous martingale with $M_{2}(0)=0$. Taking the expectation on both sides, and then using (19) and Fubini's theorem, we have

$$
\begin{aligned}
e^{\theta t} \mathbb{E}\left(x^{T}(t) Q(r(t)) x(t)\right) & \leq \lambda_{M} \mathbb{E}|x(0)|^{2}+\int_{0}^{t} e^{\theta s}\left(\theta \lambda_{M}+\lambda\right) \mathbb{E}|x(s)|^{2} d s \\
& +\int_{0}^{t} e^{\theta s} 2 \sqrt{M_{Q C K}} \mathbb{E}(|x(s)||x(s)-x(\delta(s))|) d s \\
& -\int_{0}^{t} e^{\theta s} \mathbb{E}\left[2 x^{T}(s) Q_{i} C_{i}(K(r(s))-K(r(\delta(s)))) x(\delta(s))\right] d s .
\end{aligned}
$$

Setting $a=\sqrt{\frac{2 M_{C} H_{D}\left(1-e^{-\bar{\gamma} \tau}\right)}{1-2 K(\tau)}}, b=\sqrt{\frac{2 M_{Q C K} K(\tau)}{1-2 K(\tau)}}$ and applying Lemma 3 and Lemma 4, we further derive that

$$
\begin{aligned}
& -2 \mathbb{E}\left[x^{T}(s) Q_{i} C_{i}(K(r(s))-K(r(\delta(s)))) x(\delta(s))\right] \\
\leq & a \lambda_{M} \mathbb{E}|x(s)|^{2}+\frac{\lambda_{M}}{a} \mathbb{E}\left(\left\|C_{i}\right\|^{2}\|K(r(s))-K(r(\delta(s)))\|^{2}|x(\delta(s))|^{2}\right) \\
\leq & a \lambda_{M} \mathbb{E}|x(s)|^{2}+\frac{\lambda_{M} M_{C}}{a} \mathbb{E}\left(\mathbb{E}\left(\|K(r(s))-K(r(\delta(s)))\|^{2}|x(\delta(s))|^{2} \mid \mathcal{F}_{\delta(s)}\right)\right) \\
= & a \lambda_{M} \mathbb{E}|x(s)|^{2}+\frac{\lambda_{M} M_{C}}{a} \mathbb{E}\left(|x(\delta(s))|^{2} \mathbb{E}\left(\|K(r(s))-K(r(\delta(s)))\|^{2} \mid \mathcal{F}_{\delta(s)}\right)\right) \\
= & a \lambda_{M} \mathbb{E}|x(s)|^{2}+\frac{\lambda_{M} M_{C}}{a} \mathbb{E}\left(|x(\delta(s))|^{2} \sum_{r(\delta(s))=i} \mathcal{I}_{\{r(\delta(s))=i\}} \mathbb{E}\left(\|K(r(s))-K(r(\delta(s)))\|^{2}\right)\right) \\
\leq & a \lambda_{M} \mathbb{E}|x(s)|^{2}+\frac{\lambda_{M} M_{C}}{a} \mathbb{E}\left(|x(\delta(s))|^{2} \sum_{r(\delta(s))=i} \mathcal{I}_{\{r(\delta(s))=i\}} \max _{i, j \in S}\left\|K_{j}-K_{i}\right\|^{2}\right) \\
\leq & a \lambda_{M} \mathbb{E}|x(s)|^{2}+\frac{\lambda_{M} M_{C}}{a} H_{K}\left(1-e^{-\bar{\gamma} \tau}\right) \mathbb{E}|x(\delta(s))|^{2} \\
\leq & a \lambda_{M} \mathbb{E}|x(s)|^{2}+\frac{\lambda_{M} M_{C}}{a} H_{K}\left(1-e^{-\bar{\gamma} \tau}\right)\left(2 \mathbb{E}|x(s)|^{2}+2 \mathbb{E}|x(s)-x(\delta(s))|^{2}\right) \\
\leq & a \lambda_{M} \mathbb{E}|x(s)|^{2}+\frac{\lambda_{M} M_{C}}{a} \cdot \frac{2}{1-2 K(\tau)} H_{K}\left(1-e^{-\bar{\gamma} \tau}\right) \mathbb{E}|x(s)|^{2} \\
= & 2 a \lambda_{M} \mathbb{E}|x(s)|^{2}
\end{aligned}
$$

and

$$
2 \sqrt{M_{Q C K}} \mathbb{E}(|x(s)||x(s)-x(\delta(s))|) \leq b \mathbb{E}|x(s)|^{2}+\frac{M_{Q C K}}{b} \mathbb{E}|x(s)-x(\delta(s))|^{2} \leq 2 b \mathbb{E}|x(s)|^{2} .
$$

Substituting these into (20) yields

$$
\lambda_{m} e^{\theta t} \mathbb{E}|x(t)|^{2} \leq \lambda_{M} \mathbb{E}|x(0)|^{2}+\int_{0}^{t} e^{\theta s}\left(\theta \lambda_{M}+\lambda_{\tau}\right) \mathbb{E}|x(s)|^{2} d s=\lambda_{M} \mathbb{E}|x(0)|^{2},
$$

which leads to

$$
\limsup _{t \rightarrow \infty} \frac{1}{t} \log \left(\mathbb{E}|x(t)|^{2}\right) \leq-\theta=\frac{\lambda_{\tau}}{\lambda_{M}}
$$


as required.

Let us now begin to consider the design of a feedback controller based on the discrete-time observations of both state and mode that robustly stabilizes hybrid uncertain stochastic system (1) in mean-square sense. According to Theorem 1, we need to design $K(\cdot)$, namely $K_{i}$ for $i \in S$, and further find $Q_{i}$ and positive numbers $\rho_{i}, \varepsilon_{i}$ in order for (13) and (14) to hold for any $i \in S$. Fortunately, we can transfer conditions (13) and (14) into LMIs, which it is easy to check in practice by using MATLAB Toolbox. The following theorem describes the details.

\section{Theorem 2}

Assume that there are solutions $P_{i}=P_{i}^{T}>0, \rho_{i}>0, \varepsilon_{i}>0$ and $Y_{i}(i \in S)$ to the following LMIs

$$
\Pi_{i}=\left(\begin{array}{ccccc}
\Pi_{11 i} & P_{i} N_{i}^{T} & \Pi_{31 i}^{T} & \Pi_{41 i}^{T} & \Pi_{51 i}^{T} \\
N_{i} P_{i} & -\rho_{i} I & 0 & 0 & 0 \\
\Pi_{31 i} & 0 & \Pi_{33 i} & 0 & 0 \\
\Pi_{41 i} & 0 & 0 & \Pi_{44 i} & 0 \\
\Pi_{51 i} & 0 & 0 & 0 & \Pi_{55 i}
\end{array}\right)<0,
$$

where

$$
\begin{aligned}
& \Pi_{11 i}=A_{i} P_{i}+P_{i} A_{i}^{T}+C_{i} Y_{i}+Y_{i}^{T} C_{i}^{T}+\gamma_{i i} P_{i}+\rho_{i} L_{A} L_{A}^{T}, \\
& \Pi_{33 i}=\operatorname{diag}\left(-P_{1}, \cdots,-P_{i-1},-P_{i+1}, \cdots,-P_{N}\right), \\
& \Pi_{31 i}=\left(\sqrt{\gamma_{i 1}} P_{i}, \cdots, \sqrt{\gamma_{i, i-1}} P_{i}, \sqrt{\gamma_{i, i+1}} P_{i}, \cdots, \sqrt{\gamma_{i N}} P_{i}\right)^{T}, \\
& \Pi_{44 i}=\operatorname{diag}\left(\varepsilon_{i} L_{B} L_{B}^{T}-P_{i}, \varepsilon_{i} L_{B} L_{B}^{T}-P_{i}, \cdots, \varepsilon_{i} L_{B} L_{B}^{T}-P_{i}\right), \\
& \Pi_{41 i}=\left(P_{i}\left(B_{i}^{1}\right)^{T}, P_{i}\left(B_{i}^{2}\right)^{T}, \cdots, P_{i}\left(B_{i}^{m}\right)^{T}\right)^{T}, \\
& \Pi_{55 i}=\operatorname{diag}\left(-\varepsilon_{i} I,-\varepsilon_{i} I, \cdots,-\varepsilon_{i} I\right), \\
& \Pi_{51 i}=\left(P_{i}\left(E_{i}^{1}\right)^{T}, P_{i}\left(E_{i}^{2}\right)^{T}, \cdots, P_{i}\left(E_{i}^{m}\right)^{T}\right)^{T} .
\end{aligned}
$$

Then by setting $Q_{i}=P_{i}^{-1}$ and $K_{i}=Y_{i} P_{i}^{-1}$, the controlled system (7) will be robustly exponentially stable in mean square if $\tau>0$ is small enough such that (15) holds. 


\section{Proof}

We first observe that by the well-known Schur complements (see e.g. [13]), the LMIs (21) are equivalent to the following matrix inequalities

$$
\begin{aligned}
& A_{i} P_{i}+P_{i} A_{i}^{T}+C_{i} Y_{i}+Y_{i}^{T} C_{i}^{T}+\gamma_{i i} P_{i}+\rho_{i} L_{A} L_{A}^{T} \\
+ & \rho_{i}^{-1} P_{i} N_{i}^{T} N_{i} P_{i}+\sum_{j \neq i}\left(\sqrt{\gamma_{i j}} P_{i}\right) P_{j}^{-1}\left(\sqrt{\gamma_{i j}} P_{i}\right) \\
+ & \sum_{k=1}^{m} P_{i}\left(B_{i}^{k}\right)^{T}\left(P_{i}-\varepsilon_{i} L_{B} L_{B}^{T}\right)^{-1} B_{i}^{k} P_{i}+\varepsilon_{i}^{-1} \sum_{k=1}^{m} P_{i}\left(E_{i}^{k}\right)^{T} E_{i}^{k} P_{i}<0
\end{aligned}
$$

and

$$
\varepsilon_{i} L_{B} L_{B}^{T}-P_{i}<0
$$

Recalling that $K_{i}=Y_{i} P_{i}^{-1}$ and $P_{i}=P_{i}^{T}$, we can rewrite (22) as

$$
\begin{aligned}
& A_{i} P_{i}+P_{i} A_{i}^{T}+C_{i} K_{i} P_{i}+P_{i} K_{i}^{T} C_{i}^{T}+\gamma_{i i} P_{i}+\rho_{i} L_{A} L_{A}^{T} \\
+ & \rho_{i}^{-1} P_{i} N_{i}^{T} N_{i} P_{i}+\sum_{j \neq i}\left(\sqrt{\gamma_{i j}} P_{i}\right) P_{j}^{-1}\left(\sqrt{\gamma_{i j}} P_{i}\right) \\
+ & \sum_{k=1}^{m} P_{i}\left(B_{i}^{k}\right)^{T}\left(P_{i}-\varepsilon_{i} L_{B} L_{B}^{T}\right)^{-1} B_{i}^{k} P_{i}+\varepsilon_{i}^{-1} \sum_{k=1}^{m} P_{i}\left(E_{i}^{k}\right)^{T} E_{i}^{k} P_{i}<0 .
\end{aligned}
$$

Multiplying $P_{i}^{-1}$ from left and then from right, and noting $Q_{i}=P_{i}^{-1}$, we see that the matrix inequalities (24) are equivalent to the following matrix inequalities

$$
\begin{aligned}
& Q_{i} A_{i}+A_{i}^{T} Q_{i}+Q_{i} C_{i} K_{i}+K_{i}^{T} C_{i}^{T} Q_{i}+\gamma_{i i} Q_{i}+\rho_{i} Q_{i} L_{A} L_{A}^{T} Q_{i}+\rho_{i}^{-1} N_{i}^{T} N_{i}+\sum_{j \neq i} \gamma_{i j} Q_{j} \\
& +\sum_{k=1}^{m}\left(B_{i}^{k}\right)^{T}\left(Q_{i}^{-1}-\varepsilon_{i} L_{B} L_{B}^{T}\right)^{-1} B_{i}^{k}+\varepsilon_{i}^{-1} \sum_{k=1}^{m}\left(E_{i}^{k}\right)^{T} E_{i}^{k}<0,
\end{aligned}
$$

which means the matrices in (13) are all negative-definite. Moreover, from (23) we have

$$
Q_{i}^{-1}-\varepsilon_{i} L_{B} L_{B}^{T}>0
$$

Then the required assertion follows directly from Theorem 1.

From the above theorem we can see that, to get the robust controller, we should first find solutions for (21) and then obtain small $\tau$ according to condition (15) after calculating all related quantities. Remark. As we can see, the controller in this paper is based on the discrete-time observations of both state and mode. It is more practical and costs less compared with most existing results on the same 
topic and hence our work is a useful improvement. Our controller is mode-dependent and this may be unrealistic in some real-world situations where the system mode is inaccessible. So the problem of mode-independent control for hybrid systems has attracted a lot of attention and some excellent results have appeared (see e.g. [38,39]). However, there are also some practical systems which are mode available or part available. In this case, the mode-independent results may be conservative due to the full negligence of mode information. In fact, some papers designed both mode-independent controllers and mode-dependent controllers, corresponding to the cases of mode inaccessible and mode accessible or part accessible, respectively (see e.g. [40]).

Note that on one hand, the most existing mode-independent results totally neglect system mode no matter whether it is available or not, and hence the controller $u(t)=K x(t)$ will be conservative the case where the system mode is available or part available; on the other hand, the mode-dependent controller $u(t)=K_{r(t)} x(t)$ is not applicable to the case where the system mode is inaccessible. We hence feel that we may design a new controller, like $u(t)=\left(K_{r(t)}+\rho(t) \Delta K_{r(t)}\right) x(t)\left(\Delta K_{r(t)}=\right.$ $K-K_{r(t)}$ and $\rho(t)=1$ or 0$)$, in order to combine both mode-dependent and mode-independent methods together to establish better results. This will be our further research topic.

\section{NUMERICAL EXAMPLE}

Linear hybrid stochastic differential equations have been extensively employed to model many real-world systems (see e.g. $[5,7,8]$ ). Due to the practical constraints and different limitations for different practical problems, most real-world models are very special, with constant coefficients, diagonal matrix coefficients or some other specific matrix coefficients. To illustrate the theory developed in this paper better, we will use more general system matrices instead of diagonal matrices etc.

Let us consider a two-dimensional linear hybrid uncertain SDE

$$
d x(t)=[A(r(t))+\Delta A(t, r(t))] x(t) d t+[B(r(t))+\Delta B(t, r(t))] x(t) d w(t)
$$


on $t \geq 0$. Here $w(t)$ is a scalar Brownian motion and $r(t)$ is a Markov chain on the state space $S=\{1,2\}$ with the generator

$$
\Gamma=\left[\begin{array}{rr}
-3 & 3 \\
2 & -2
\end{array}\right]
$$

$\Delta A(t, r(t))$ and $\Delta B(t, r(t))$ are defined as in (3) and (4) and all the other matrices are given by

$$
\begin{gathered}
A_{1}=\left[\begin{array}{rr}
-2.1 & 0.1 \\
0.1 & 1.1
\end{array}\right], \quad A_{2}=\left[\begin{array}{rr}
-1.9 & -0.1 \\
-0.1 & 0.9
\end{array}\right], \quad L_{A}=\left(\begin{array}{l}
0.1 \\
0.1
\end{array}\right), \\
B_{1}=\left[\begin{array}{rr}
0.2 & 1 \\
0 & 0.2
\end{array}\right], \quad B_{2}=\left[\begin{array}{rr}
0.1 & 0 \\
0 & 0.3
\end{array}\right], \quad L_{B}=\left(\begin{array}{l}
0.2 \\
0.2
\end{array}\right), \\
N_{1}=\left(\begin{array}{ll}
0.2 & 0.1
\end{array}\right), \quad N_{2}=\left(\begin{array}{ll}
0.13 & 0.21
\end{array}\right) \\
E_{1}=\left(\begin{array}{ll}
0.4 & 0.3
\end{array}\right), \quad E_{2}=\left(\begin{array}{ll}
0.24 & 0.31
\end{array}\right) .
\end{gathered}
$$

The numerical simulation (Figure 5.1) shows that this hybrid uncertain SDE is not almost surely exponentially stable (and then certainly not mean-square exponentially stable as the latter implies the former).

Now we aim to design a feedback control based on discrete-time observations of both state and mode to stabilize the system. Assume that the controlled linear hybrid uncertain SDE has the form

$$
\begin{aligned}
d x(t) & =[(A(r(t))+\Delta A(t, r(t))) x(t)+C(r(t)) K(r(\delta(t))) x(\delta(t))] d t \\
& +[B(r(t))+\Delta B(t, r(t))] x(t) d w(t),
\end{aligned}
$$

where

$$
C_{1}=\left(\begin{array}{c}
0.1 \\
0.7
\end{array}\right), \quad C_{2}=\left(\begin{array}{c}
0.2 \\
0.5
\end{array}\right) .
$$

Our aim is to find $K_{1}$ and $K_{2}$ in $R^{1 \times 2}$ and then make sure $\tau$ is sufficiently small for this controlled SDE to be exponentially stable in mean square. To apply Theorem 2, we first find that LMIs (21) have the feasible solutions

$$
P_{1}=\left[\begin{array}{ll}
8.8284 & 0.0897 \\
0.0897 & 8.5196
\end{array}\right], \quad P_{2}=\left[\begin{array}{cc}
9.0255 & 0.1382 \\
0.1382 & 9.4558
\end{array}\right],
$$



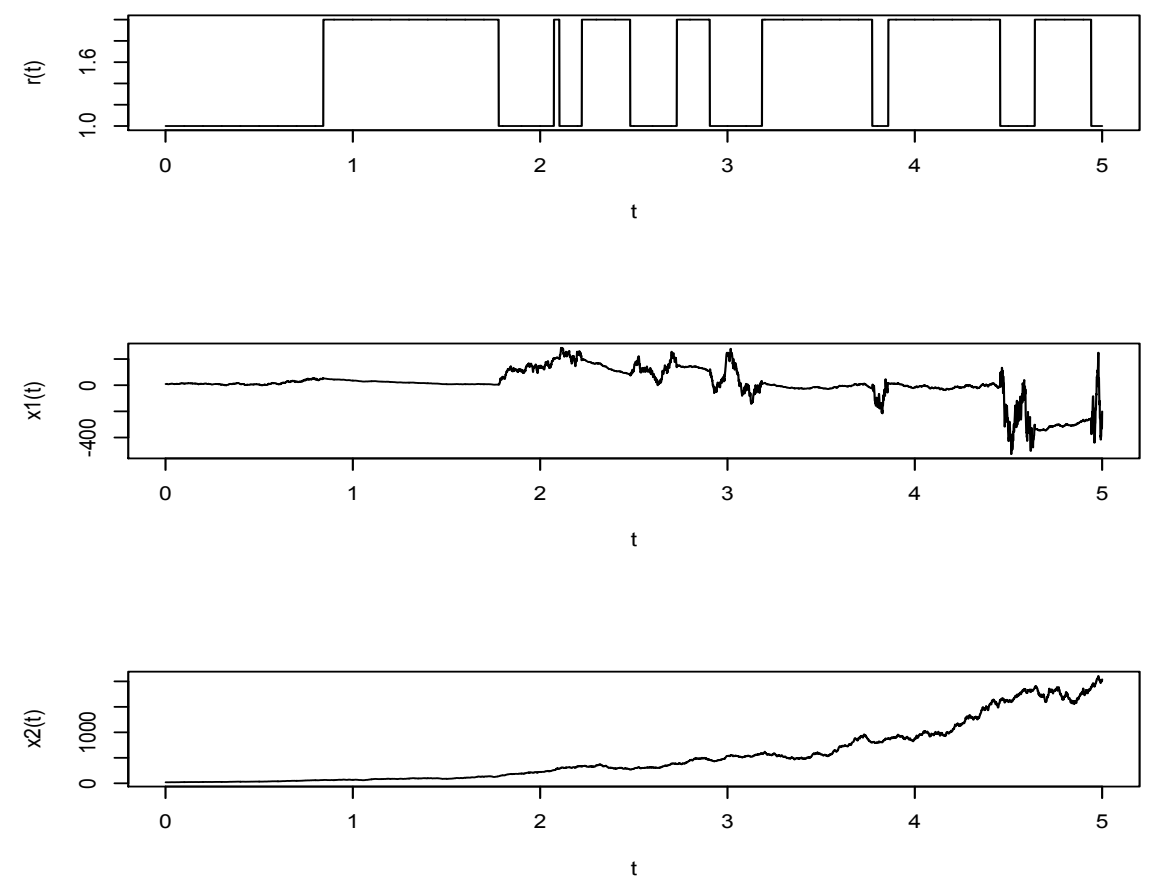

Fig. 5.1. Computer simulation of the paths of $r(t), x_{1}(t)$ and $x_{2}(t)$ for the hybrid uncertain SDE (26) using the Euler-Maruyama method with step size $10^{-6}$ and initial values $r(0)=1, x_{1}(0)=10$ and $x_{2}(0)=20$.

$$
\begin{gathered}
Y_{1}=\left(\begin{array}{ll}
3.9907 & -30.7673
\end{array}\right), \quad Y_{2}=\left(\begin{array}{ll}
23.5315 & -40.1093
\end{array}\right), \\
\rho_{1}=21.8561, \quad \rho_{2}=21.6545, \quad \varepsilon_{1}=18.0638, \quad \varepsilon_{2}=18.8368 .
\end{gathered}
$$

A further calculation shows that

$$
K_{1}=\left(\begin{array}{ll}
0.4888 & -3.6165
\end{array}\right), \quad K_{2}=\left(\begin{array}{ll}
2.6728 & -4.2808
\end{array}\right)
$$

and

$$
\begin{gathered}
\lambda_{M}=0.1177, \quad \lambda_{m}=0.1053, \quad \lambda=-0.2384, \\
M_{Q C K}=0.0911, \quad M_{C}=0.5, \quad H_{K}=5.2112, \\
M_{A}=8.8482, \quad M_{C K}=7.3861, \quad M_{B}=2.1970 .
\end{gathered}
$$


It is easy to show that (15) holds whenever $\tau<0.0031$. So by Theorem 2, if we set $K_{i}(i=1,2)$ as above and make sure $\tau<0.0031$, then the controlled system (27) is mean-square exponentially stable. The numerical simulation (Figure 5.2) supports this result clearly.
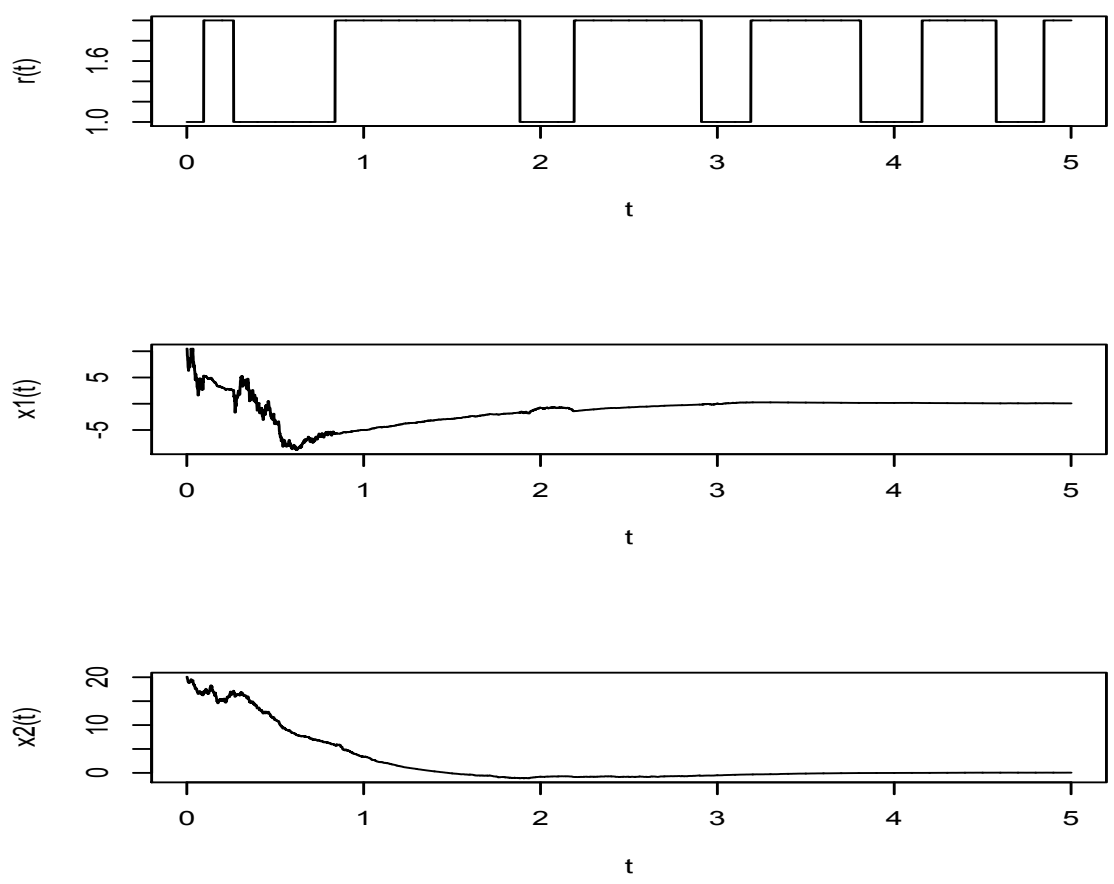

Fig. 5.2. Computer simulation of the paths of $r(t), x_{1}(t)$ and $x_{2}(t)$ for the controlled hybrid uncertain SDE (27) with $\tau=0.002$ using the Euler-Maruyama method with step size $10^{-6}$ and initial values

$$
r(0)=1, x_{1}(0)=10 \text { and } x_{2}(0)=20 .
$$

\section{CONCLUSION}

In this paper, we have showed that unstable hybrid uncertain stochastic systems can be stabilized by linear state feedback controls based on the discrete-time observations of both state and mode. Such discrete-time controls are more realistic and cost less compared with continuous-time feedback controls. A method in terms of LMIs for designing the robust controller has also been developed. An numerical example is provided to demonstrate the effectiveness of our theory. 


\section{ACKNOWLEDGEMENTS}

The authors would like to thanks the reviewers and editor for their very professional comments and suggestions which have significantly improved this paper. This paper was completed when the first author was visiting the Department of Mathematics and Statistics, University of Strathclyde, whose hospitality is greatly appreciated. The authors would like to thank the financial support from Donghua University (15D310414) and the Leverhulme Trust (RF-2015-385), and the first author would especially like to thank the Chinese Scholarship Council for awarding her the scholarship to visit the University of Strathclyde for one year.

\section{REFERENCES}

1. Kushner H. Stochastic Stability and Control. Academic Press: New York, 1976.

2. Khasminskii RZ. Stochastic Stability of Differential Equations. Sijthoff and Noordhoff: Leiden, 1981.

3. Mao X. Stochastic Differential Equations and Applications (Second Edition). Horwood Publishing Limited: Chichester, 2007.

4. Mohammed SEA. Stochastic Functional Differential Equations. Longman Scientific and Technical: Harlow, 1986.

5. Ghosh MK, Arapostathis A, Marcus SI. Optimal control of switching diffusions with application to flexible manufacturing systems. SIAM J. Control Optim. 1993; 31(5):1183-1204.

6. Ghosh MK, Arapostathis A, Marcus SI. Ergodic control of switching diffusions. SIAM J. Control Optim. 1997; 35(6):1952-1988.

7. Yin G, Liu RH, Zhang Q. Recursive algorithms for stock liquidation: A stochastic optimization approach. SIAM J. Optim. 2002; 13(1):240-263.

8. Mariton M. Jump Linear Systems in Automatic Control. Marcel Dekker: New York, 1990.

9. Basak GK, Bisi A, Ghosh MK. Stability of a random diffusion with linear drift. J. Math. Anal. Appl. 1996; 202:604622.

10. Mao X. Stability of stochastic differential equations with Markovian switching. Sto. Proc. Their Appl. 1999; 79:4567.

11. Mao X. Exponential stability of stochastic delay interval systems with Markovian switching. IEEE Trans. Auto. Control 2002; 47(10):1604-1612.

12. Yue D, Han Q. Delay-dependent exponential stability of stochastic systems with time-varying delay, nonlinearity, and Markovian switching. IEEE Trans. Automat. Control 2005; 50:217-222.

13. Mao X, Yuan C. Stochastic Differential Equations with Markovian Switching. Imperial College Press: London, 2006. 
14. Mao X, Yin G, Yuan C. Stabilization and destabilization of hybrid systems of stochastic differential equations. Automatica 2007; 43:264-273.

15. Mao X, Lam J, Huang L. Stabilisation of hybrid stochastic differential equations by delay feedback control. Systems \& Control Letters 2008; 57:927-935.

16. Wu L, Su X, Shi P. Sliding mode control with bounded $L_{2}$ gain performance of Markovian jump singular time-delay systems. Automatica 2012; 48(8):1929-1933.

17. Zhang LX, Ning ZP, Shi P. Input-output approach to control for fuzzy Markov jump systems with time-varying delays and uncertain packet dropout rate. IEEE Transactions on Cybernetics 2015; 45(11):2449-2460.

18. Zhang LX, Zhu YZ, Shi P, Zhao YX. Resilient asynchronous $H_{\infty}$ filtering for Markov jump neural networks with Unideal measurements and multiplicative noises. IEEE Transactions on Cybernetics 2015; 45(12):2840-2852.

19. Chen W, Guan Z, Lu X. Delay-dependent exponential stability of uncertain stochastic systems with multiple delays: an LMI approach. Systems Control Lett. 2005; 54:547-555.

20. Niu YG, Ho DWC, Lam J. Robust integral sliding mode control for uncertain stochastic systems with time-varying delay. Automatica $2005 ;$ 41:873-880.

21. Shi P, Mahmoud MS, Yi J, Ismail A. Worst case control of uncertain jumping systems with multi-state and input delay information. Information Sciences 2006; 176:186-200.

22. Wei G, Wang Z, Shu H, Fang J. Robust $H_{\infty}$ control of stochastic time-delay jumping systems with nonlinear disturbances. Optim. Control Appl. Meth. 2006; 27:255-271.

23. Xu S, Lam J, Wang JL, Yang GH. Stabilization and $H_{\infty}$ control for uncertain stochastic time-delay systems via non-fragile controllers. Asian Journal of Control 2006; 8:197-200.

24. Moon YS, Park P, Kwon WH, Lee YS. Delay-dependent robust stabilization of uncertain state-delayed systems. International Journal of Control 2001; 74(14):1447-1455.

25. Yue D, Han QL. Delayed feedback control of uncertain systems with time-varying input delay. Automatica 2005; 41(2):233-240.

26. Lu CY, Tsai JSH, Jong GJ, Su TJ. An LMI-based approach for robust stabilization of uncertain stochastic systems with time- varying delays. IEEE Trans. Automat. Control 2003; 48(2):286-289.

27. Wang Z, Qiao H, Burnham KJ. On stabilization of bilinear uncertain time-delay stochastic systems with Markovian jumping parameters. IEEE Trans. Automat. Control 2002; 47(4):640-646.

28. Huang L, Mao X. Robust delayed-state-feedback stabilization of uncertain stochastic systems. Automatica 2009; 45:1332-1339.

29. Mao X. Stabilization of continuous-time hybrid stochastic differential equations by discrete-time feedback control. Automatica 2013; 49(12):3677-3681

30. Allwright JC, Astolfi A, Wong HP. A note on asymptotic stabilization of linear systems by periodic, piecewise constant, output feedback. Automatica 2005; 41(2):339-344.

31. Hagiwara T, Araki M. Design of stable state feedback controller based on the multirate sampling of the plant output. IEEE Trans. Automat. Control 1988; 33(9):812-819. 
32. Hagiwara T, Araki M. On preservation of strong stabilizability under sampling. IEEE Trans. Automat. Control 1988; 33(11):1080-1082.

33. Mao X, Liu W, Hu L, Luo Q, Lu J. Stabilization of hybrid SDEs by feedback control based on discrete-time state observations. Systems \& Control Letters 2014; 73:88-95.

34. You S, Liu W, Lu J, Mao X, Qiu Q. Stabilization of hybrid systems by feedback control based on discrete-time state observations. SIAM J. Control Optim. 2015; 53(2):905-925.

35. Geromel JC, Gabriel GW. Optimal $H_{2}$ state feedback sampled-data control design of Markov jump linear systems. Automatica 2015; 54:182-188.

36. Wang Y, Xie L, De Souza CE. Robust control of a class of uncertain nonlinear systems. Systems \& Control Letters 1992; 19:139-149.

37. Anderson WJ. Continuous-Time Markov Chains. Springer: New York, 1991.

38. Pan GL, Bar-Shalom Y. Stabilization of jump linear Gaussian systems without mode observations. Int. J. Control 1996; 64(4):631-661.

39. Wu HN, Cai KY. Mode-independent robust stabilization for uncertain Markovian jump nonlinear systems via fuzzy control. IEEE Transactions on Systems, Man and Cybernetics-Part B: Cybernetics 2006; 36(3):509-519.

40. De Souza CE. Robust stability and stabilization of uncertain discrete-time Markovian jump linear systems. IEEE Trans. Automat. Control 2006; 51(5):836-841. 